\title{
RD50 Recent Results - Development of radiation hard sensors for SLHC
}

\author{
Anna Macchiolo \\ Max-Planck-Institut für Physik \\ Föhringer Ring 6, Munich, Germany \\ E-mail: Anna.Macchiolo@mppmu.mpg.de
}

\section{on behalf of the RD50 Collaboration}

The need for radiation hard semiconductor detectors for the tracker regions in high energy physics experiments at a future high luminosity hadron collider, like the proposed LHC upgrade, has led to the formation of the CERN RD50 collaboration. The R\&D directions of RD50 follow two paths: the optimization of radiation hard bulk materials (Material Engineering) and the development of new detector designs (Device Engineering) as 3D sensors, thin sensors and n-in-p sensors.

Some of the RD50 most recent results about silicon detectors are reported in this paper, with special reference to: (i) identification of defects responsible for long term annealing, (ii) charge collection efficiency of irradiated planar devices, in particular n-in-p microstrip detectors and epitaxial diodes, (iii) charge collection efficiency of double-type column 3D detectors, (iv) comparison of the performances of FZ and MCZ structures under mixed irradiation.

VERTEX 2009 (18 ${ }^{\text {th }}$ workshop) - VERTEX 2009

Veluwe, the Netherlands

September 13-18, 2009 


\section{Introduction}

An upgrade of the LHC accelerator (Super-LHC, SLHC) is foreseen to increase the instantaneous luminosity by a factor of ten with respect to the present design luminosity of $10^{34}$ $\mathrm{cm}^{-2} \mathrm{~s}^{-1}$. The upgrade process will be divided in two stages: Phase- 1 will deliver a luminosity of (2-3) $\times 10^{34} \mathrm{~cm}^{-2} \mathrm{~s}^{-1}$ with changes mainly restricted to the injection system and to the inner triplets of the interaction regions whereas Phase-2 will reach a maximum luminosity of $10^{35} \mathrm{~cm}^{-2} \mathrm{~s}^{-1}$ with the modification of several machine parameters. In this scenario the innermost layers of the tracking detectors of the ATLAS and CMS experiments will have to sustain fluences reaching (3-4) $\times 10^{15} \mathrm{n}_{\mathrm{eq}} / \mathrm{cm}^{2}$ during the Phase-1 upgrade and (1-2) $\times 10^{16} \mathrm{n}_{\mathrm{eq}} / \mathrm{cm}^{2}$ during the Phase-2 running period. Upgrades restricted to the pixel layers are foreseen in view of Phase-1 for the tracking systems, while their complete replacement will be necessary to sustain the Phase-2 radiation environment. The upgrade of the LHCb VErtex LOcator also poses similar challenges with a total fluence at the end of the upgrade lifetime expected to be $0.8 \times 10^{16} \mathrm{n}_{\mathrm{eq}} / \mathrm{cm}^{2}$ [1]. The aim of the RD50 Collaboration [2] is the development of radiation-hard semiconductor sensors to satisfy the SLHC upgrade requirements. The approach followed by the RD50 Collaboration consists in pursuing two major research lines: optimization of radiation hard bulk materials (Material Engineering) and development of new detector designs (Device Engineering) as 3D sensors, thin sensors and n-in-p sensors.

In this paper a selection of recent results obtained by the RD50 Collaboration is presented.

\section{Defect and material characterization}

The radiation induced degradation of the sensor performance is mainly due to defects of the silicon bulk.
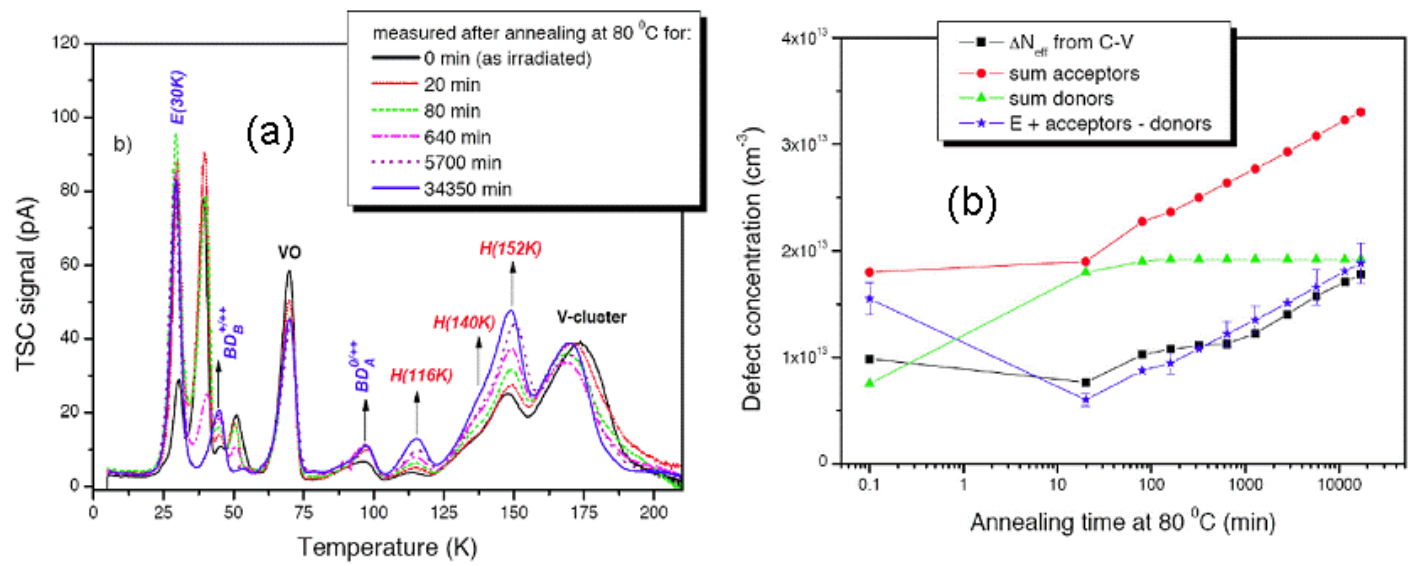

Fig. 1: (a) Spectrum obtained by means of the Thermally Stimulated Currents (TSC) technique for different isothermal annealing steps at $80^{\circ} \mathrm{C}$ of an epitaxial silicon diode irradiated with $23 \mathrm{GeV}$ protons at a fluence of $2.3 \times 10^{14} \mathrm{n}_{e q} / \mathrm{cm}^{2}$. (b) Defect concentration as a function of the annealing time extracted from CV characteristics and TSC scans. Data are taken from [3]. 
The understanding of the creation mechanism for these defects and their effect on the macroscopic sensor properties pave the way to possible improvements of the detector performance through material engineering.

Recently many efforts have been spent to study the so called "cluster" related defects, densely packed conglomerates of vacancies and interstitial defects produced in the silicon bulk by hadrons and high energetic electrons [3]. One of the major breakthroughs in this field has been the identification of the deep acceptors $\mathrm{H}(116 \mathrm{~K}), \mathrm{H}(140 \mathrm{~K})$ and $\mathrm{H}(152 \mathrm{~K})$ and the shallow donor $\mathrm{E}(30 \mathrm{~K})$ as the responsible defects for the long term annealing behaviour of the space charge concentration [4]. They were firstly detected by means of the Thermally Stimulated Currents (TSC) technique after hadron irradiation but are not observed after ${ }^{60} \mathrm{Co} \gamma$-irradiation, where the maximal silicon recoil energy is too low for the creation of defect clusters. The experimental procedure consists in first filling the energy levels either by optical or electrical injection, usually at a relatively low temperature. Subsequently the sample is heated under reverse bias and at a specified temperature the trapped charges (electrons or holes) are emitted giving rise to a unique current peak signal for each kind of defect level. The defect concentration can be calculated from the total charge released from the defects during heating.

As seen in Fig. 1a, these defect concentrations are increasing with annealing time. In Fig. $1 \mathrm{~b}$ the effective doping concentration as measured by CV characteristics is compared to the sum of the positive space charge concentration of the $\mathrm{E}(30 \mathrm{~K})$ defect and the negative space charge concentration of the $\mathrm{H}$ centers, as obtained by the TSC technique. Good agreement is observed as a function of the annealing time, proving the dominant role of the $\mathrm{H}$ centers and the $\mathrm{E}(30 \mathrm{~K})$ defect in determining the evolution of the space charge concentration or depletion voltage during the annealing process.

\section{Device engineering}

The use of p-type silicon sensors, with strip or pixel devices, has been intensively studied by the RD50 Collaboration. Also after heavy irradiations the junction and high field region remains at the signal collection electrodes, improving charge collection time and efficiency (CCE) [5]. Furthermore these sensors can be operated partially depleted. Since such a configuration collects electrons instead of holes it is also less affected by trapping. Fig. 2 summarizes the results of several CCE measurements performed on Float Zone (FZ) strip sensors as a function of the received fluence at different bias voltages. It is clear that p-in-n sensors used in the present trackers of the LHC experiments are not able to supply a sufficient charge after having been irradiated to SLHC fluences.

On the other hand, these results show, that even at fluences of $10^{16} \mathrm{n}_{\mathrm{eq}} / \mathrm{cm}^{2}$, n-in-p sensors yield signals around 6000-7000 electrons for bias voltages of $800 \mathrm{~V}$. It is therefore possible to observe higher signals than expected from charge trapping models based on measurements performed after lower fluences. Simulations [6] have shown that neither a reduced acceptor introduction nor a non-linear scaling of the trapping probability can explain the observed charge collection. 
Measurements at high bias voltages on $140 \mu \mathrm{m}$ thick n-in-p strip sensors, irradiated to a fluence of $5 \times 10^{15} \mathrm{n}_{\mathrm{eq}} / \mathrm{cm}^{2}$, have achieved collected charges that are clearly in excess of the ones obtained for unirradiated sensors [7].

Recent results [8] obtained with the Edge-TCT technique on n-in-p strip sensors, irradiated up to a fluence of $5 \times 10^{15} \mathrm{n}_{\mathrm{eq}} / \mathrm{cm}^{2}$, substantiate the explanation of the increased charge collection by an avalanche multiplication process taking place in the high electric field region near the electrodes.

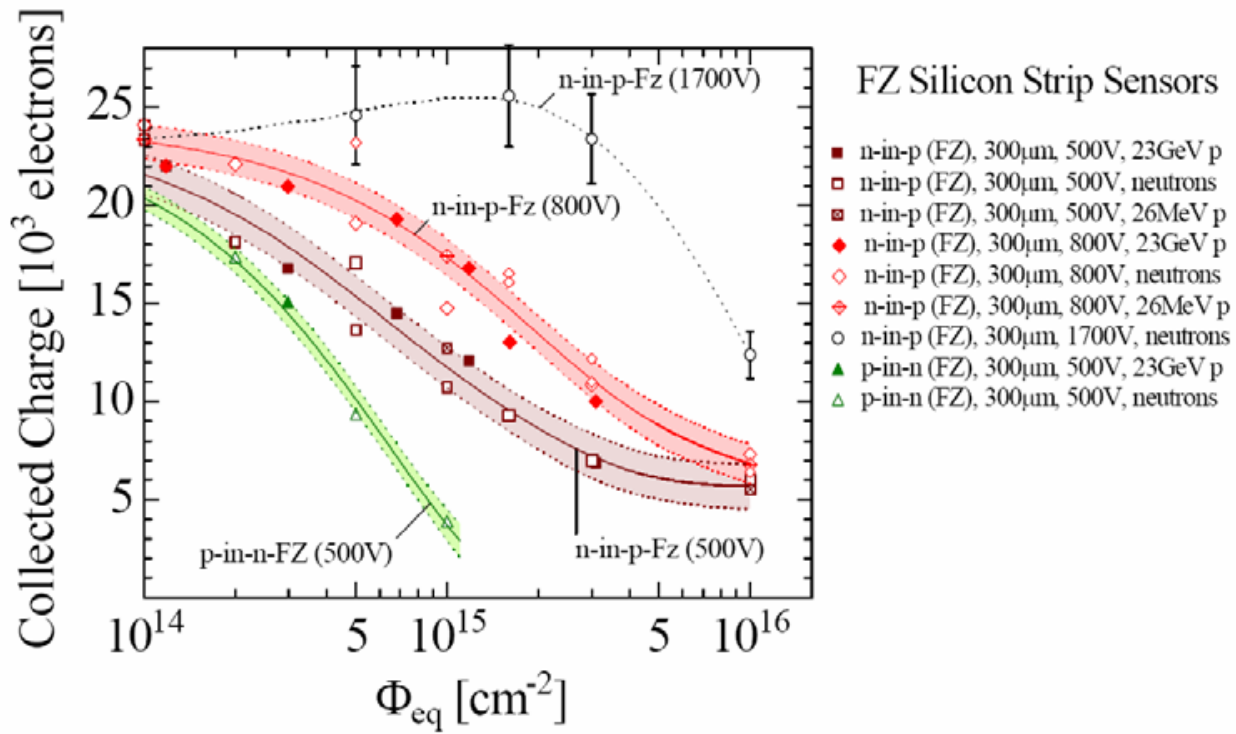

Fig. 2: Collected Charge in FZ strip sensors as a function of the received fluence after neutron and proton irradiations. Measurements have been performed between $-20^{\circ} \mathrm{C}$ and $-40^{\circ} \mathrm{C}$. Data are taken from $[9,10]$

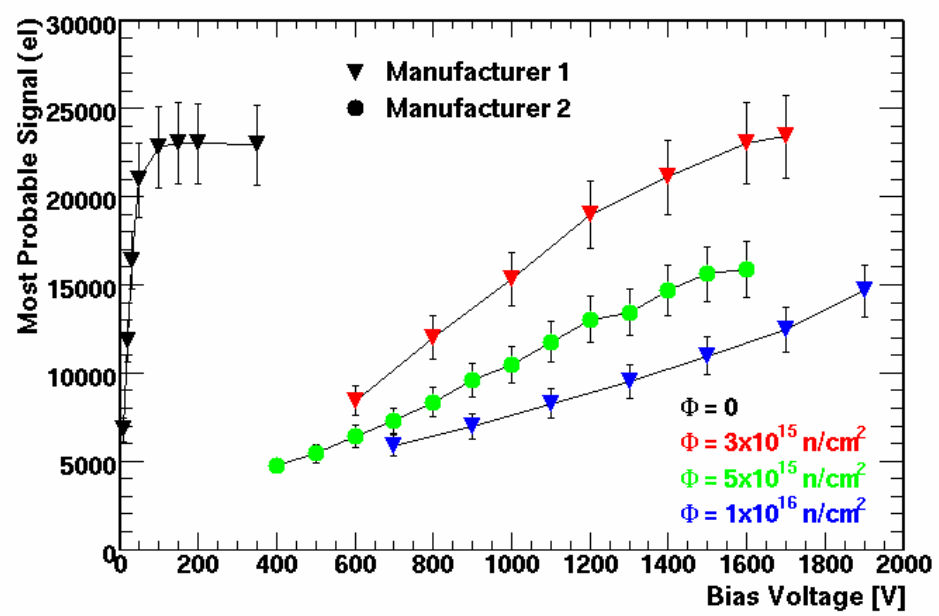

Fig. 3: Charge collection efficiency as a function of the bias voltage for the Hamamatsu strip sensor (Manufacturer 2) irradiated to $5 \times 10^{15} \mathrm{n}_{e q} / \mathrm{cm}^{2}$. Superimposed are measurements of Micron detectors (Manufacturer 1) irradiated to $3 \times 10^{15}$ and $1 \times 10^{16} \mathrm{n}_{e q} / \mathrm{cm}^{2}$. 
Most of the devices used in the measurements reported in Fig. 2 have been fabricated by Micron ${ }^{1}$. The charge multiplication mechanism has been recently confirmed also by measurements of strip sensors produced by $\mathrm{HPK}^{2}$ [11]. Fig. 3 shows that, after having taken into account the corresponding fluences, the behavior of the HPK sensor in terms of charge collection efficiency is consistent with the one observed in the Micron devices.

An anomalous high collected charge has also been observed with measurements performed on n-type epitaxial diodes, 75 to $150 \mu \mathrm{m}$ thick [12]. As shown in Fig. 4a, the CCE at lower fluences exhibits a saturation behavior whereas the CCE of highly irradiated diodes increases rapidly at high bias voltages and exceeds clearly a value of 1 . The effect is more relevant for the $75 \mu \mathrm{m}$ thick diodes than for the $150 \mu \mathrm{m}$ thick ones, due to a reduced drift distance and a higher electric field resulting for the same bias voltage applied. Fig. $4 \mathrm{~b}$ compares the CCE measurements obtained with various methods, as a function of voltage, to the IV characteristics of the sensor, expressed in arbitrary units. The CCE, obtained with laser lights of different wavelengths or a $5.8 \mathrm{MeV} \alpha$-particles source, is higher in case of a smaller penetration depth, pointing to the conclusion that the charge multiplication mechanism is taking place in the first few microns under the collecting electrode. Moreover, the voltage dependence of the collected charge induced by a $670 \mathrm{~nm}$ laser and of the leakage current is almost identical.
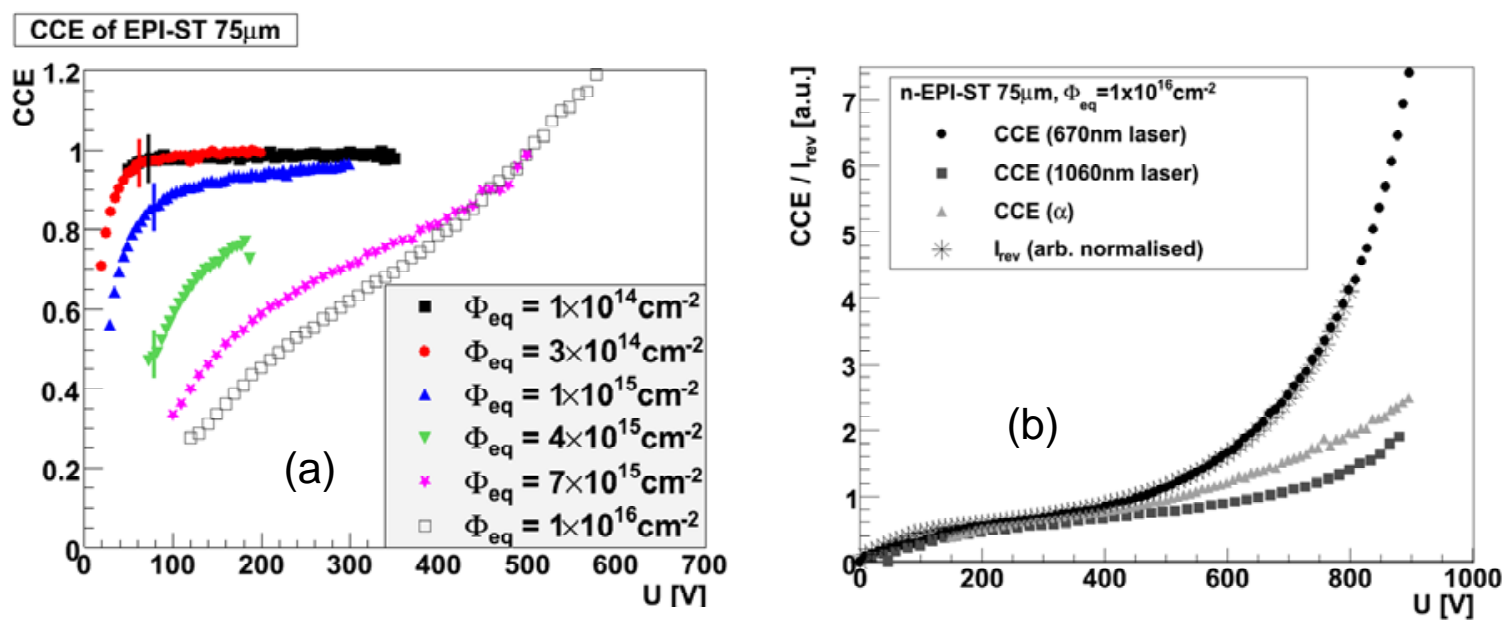

Fig. 4: (a) CCE, obtained with a $5.8 \mathrm{MeV} \alpha$-particle source, measured as a function of the voltage in $n$ type epitaxial diodes, $75 \mu \mathrm{m}$ thick, irradiated at different fluences. (b) Comparison of the CCE dependence on the voltage, as obtained with different methods, with the IV curve of the corresponding sensor. The leakage current values are expressed in arbitrary units. The CCE curve relative to the 670 nm laser and the IV curve are almost superimposed.

\subsection{Planar pixel productions}

The results previously quoted supply a strong motivation to investigate the use of planar pixel devices as possible candidate detectors to instrument the inner layers of the pixel systems at SLHC. Within the framework of the RD50 Collaboration different pixel productions have been completed or are under way.

\footnotetext{
${ }^{1}$ Micron Semiconductor Ltd., http://www.micronsemiconductor.co.uk

${ }^{2}$ Hamamatsu Photonics, K.K. http://www.hamamatsu.com
} 
Exploring the properties of thin planar sensors is particularly appealing for the reduction in the associated radiation length and for the higher electric field that can lead to an enhanced signal size after irradiation through the charge multiplication effect. A first production of thin nin-p planar pixel sensors has been completed with an active thickness of $75 \mu \mathrm{m}$ and $150 \mu \mathrm{m}$ exploiting a wafer bonding technology that allows to freely tuning both the active and the total thickness of the device [13].

The pre-irradiation characterization of the devices has been carried out, resulting in a good yield ( 98\%) and leakage currents lower than $10 \mathrm{nA} / \mathrm{cm}^{2}$, as shown in Fig. 5 [14].

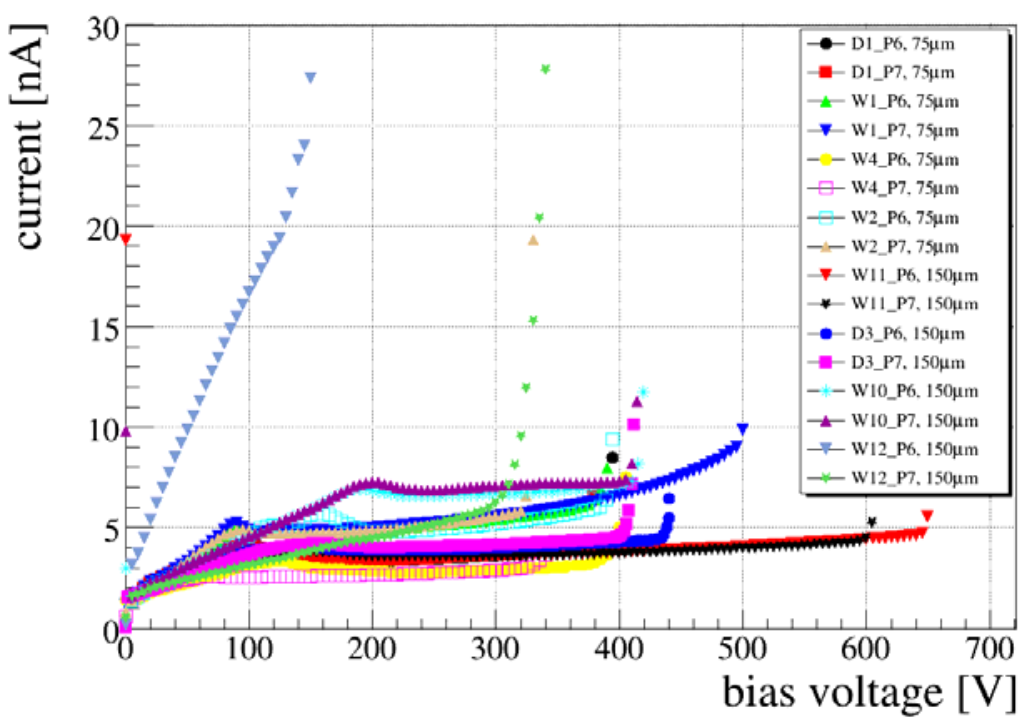

Fig.5: Leakage current characteristics of n-in-p pixel detectors, with an active thickness of $75 \mu \mathrm{m}$ and $150 \mu \mathrm{m}$ and geometry compatible with a single chip of the present ATLAS pixel electronics. The first entry in the legend indicates the wafer number, the second one the sensor position in the wafer and the third one the active thickness

\subsection{D detectors}

3D sensors are promising candidates to instrument the inner layers of the tracker systems at SLHC. In the original conception of this kind of devices, holes are etched into the silicon bulk with a matrix arrangement and they are then alternating filled with polysilicon $n$ - and p-type doped. By applying a bias voltage between the differently doped columns the bulk is depleted horizontally and the charge generated is collected by the columns. The decoupling of the sensor thickness from the charge collection distance is of great advantage for irradiated sensors because the shorter distance between electrodes leads to a reduced $V_{f d}$ and lower charge trapping. Within the RD50 Collaboration simplified versions of the original and challenging 3D design are under study. The Double-Side Double-Type Column (DDTC) 3D design features columnar electrodes of one type being processed from the front surface of the detector and electrodes of the other type from the back side $[15,16]$. Furthermore, the columns do not pass through the full thickness of the substrate, which makes the devices more resistant to mechanical stress than full 3D detectors, and avoids the necessity of a carrier wafer. 
Unirradiated DDTC devices, produced by $\mathrm{FBK}^{3}$ and $\mathrm{CNM}^{4}$, have been tested in a high energy pion beam at CERN. A uniform charge collection efficiency has been observed, with the exception of the electrode positions, due to the beam being parallel to the columns [17]. Full charge collection efficiency for the unirradiated devices has been proved both during the beam test and in response to radioactive sources [18,19]

\section{Mixed irradiation experiments}

Within RD50 a program of mixed irradiations has started to reproduce more realistically the radiation conditions of the tracking detectors at SLHC [20]. Here the charged hadrons represent the dominant contribution at smaller radii while the neutrons originating in the calorimeters become the most relevant radiation source from a radius of $25 \mathrm{~cm}$ outwards. It was already known that under charged particle irradiation n-type magnetic Czochralski (MCZ) and n- and p-type epitaxial silicon behave differently than FZ silicon because, in addition to the negatively charged acceptors generated in all cases, in the oxygen-rich silicon materials the creation of positively-charged shallow donors dominates.
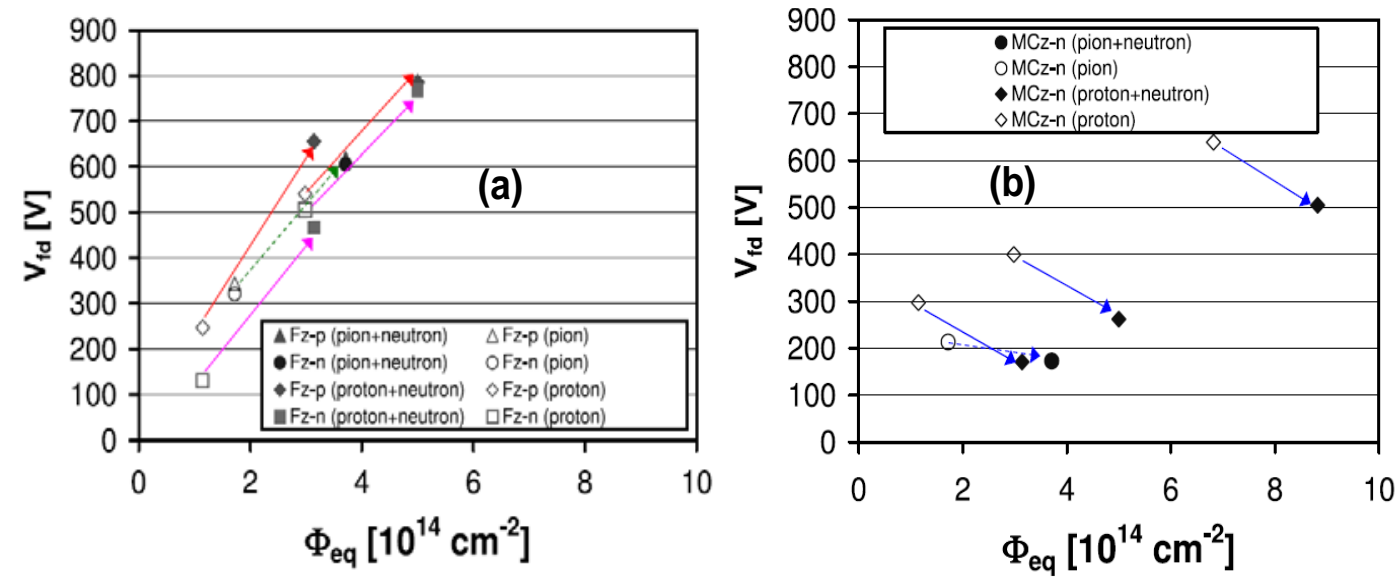

Fig. 6: Dependence of $V_{f d}$ on the equivalent fluence for mixed irradiated samples: (a) FZ n- and p-type sensors, (b) n-type MCZ sensors. The arrows denote the direction of change for $V_{f d}$ from control to mixed irradiated samples.

Fig. 6 shows the dependence of $\mathrm{V}_{\mathrm{fd}}$ for FZ (left) and MCZ (right) sensors after irradiations with either $23 \mathrm{GeV}$ protons or $200 \mathrm{MeV}$ pions and subsequently with a fluence of $2 \times 10^{14}$ $\mathrm{n}_{\mathrm{eq}} / \mathrm{cm}^{2}$ of neutrons. The reference sample did not undergo the neutron irradiation. In the $\mathrm{FZ}$ detectors the neutron irradiation causes the introduction of additional effective acceptors with respect to the reference sample and an increase of $V_{\mathrm{fd}}$. In the n-type MCZ silicon instead, $V_{\mathrm{fd}}$ is seen to decrease after neutron irradiation, proving that the dominant space charge after charged hadron irradiation up to the maximum tested fluence of $6.8 \times 10^{14} \mathrm{n}_{\mathrm{eq}} / \mathrm{cm}^{2}$ is still positive due to the dominant contributions of shallow donors. The compensation effect of the different kind of irradiations has also been observed with CCE measurements on FZ and MCZ n-in-n strip sensors, irradiated in a first step with protons at a fluence of $5 \times 10^{14} \mathrm{n}_{\mathrm{eq}} / \mathrm{cm}^{2}$. After a subsequent

\footnotetext{
${ }^{3}$ Fondazione Bruno Kessler - FBK, Povo, Trento, Italy, http://memsrad.fbk.eu/en/home

${ }^{4}$ Centro Nacional de Microelectrónica (IMB-CNM, CSIC), Barcelona, Spain, http://www.cnm.es
} 
irradiation with neutrons of the same equivalent fluence, the FZ sensors exhibit a decrease of the collected charge while the MCZ sensors yield a higher signal size [9]. It is foreseen to extend these studies to different mixtures of charged hadrons and neutrons irradiations.

\section{Conclusions}

A review of recent salient results of the RD50 Collaboration has been presented.

In the field of defect and material characterization a major finding has been the identification of the defects responsible for the reverse annealing in the case of hadron-induced damage. Regarding the activities connected to the Device Engineering, FZ n-in-p sensors have been shown to yield enough charge to be used for the strip detectors in the external layers of the upgraded trackers at SLHC. At very high voltages and fluences, planar n-in-p detectors exhibit an increased signal with respect to the predictions of charge trapping models based on measurements performed at lower fluences. Recent results have led to the interpretation of this phenomenon as a charge multiplication effect, which likely is taking place in the high electric field near the electrodes. Several studies are being carried out within the RD50 Collaboration to improve the understanding of the charge multiplication mechanism and possibly tune the sensor production parameters to fully exploit its potentials. Planar sensors can be regarded, in view of these results, as good candidates to instrument also the inner pixel layers at SLHC.

The other promising option is represented by 3D detectors, which, within the RD50 Collaboration, are investigated in the DDTC version. Also in the case of this modified architecture, aimed at a simplification of the manufacturing technology, full charge collection efficiency in unirradiated sensors has been proved with beam tests and measurements with radioactive sources.

\section{References}

[1] P. Collins et al., "The LHCb VELO Upgrade”, Proceedings of the HSTD7 Symposium, Hiroshima (Japan), 2009.

[2] http://www.cern.ch/rd50

[3] I. Pintilie et al., Nucl. Instr. Meth. A 611 (2009) 52-68.

[4] I. Pintilie et al., Appl. Phys. Lett. 92 (2008) 024101.

[5] G. Casse et al., Nucl. Instr. Meth. A 535 (2004) 362.

[6] I. Mandic et al., Nucl. Instr. Meth. A (2009) in press, doi:10.1016/j.nima.2009.08.004

[7] G. Casse et al., "Enhanced efficiency of segmented silicon detectors of different thicknesses after proton irradiations up to $1 \times 10^{16} \mathrm{n}_{\mathrm{eq}} \mathrm{cm}^{-2}$, , presented at the $11^{\text {th }}$ ESSD, Wildbad Kreuth, 2009, accepted in Nucl. Instr. Meth. A.

[8] G. Kramberger et al., "Charge Collection Properties of Heavily Irradiated Silicon Micro-strip Detectors Studied by Edge Transient Current-Technique (E-TCT)", Conference Report of the IEEE NSS-MIC Conference, Orlando (USA), 2009. 
[9] G. Casse et al., "Measurements of charge collection efficiency with micro-strip detectors made on various substrates after irradiations with neutrons and protons with different energies”, Proceedings of Science, PoS(Vertex2008) 036.

[10] I. Mandic et al., Nucl. Instr. Meth. A 603 (2009) 263.

[11] M. Mikuz et al., "Study of Anomalous Charge Collection Efficiency in Heavily Irradiated Silicon Strip Detectors”, Proceedings of the HSTD7 Symposium, Hiroshima (Japan) , 2009.

[12] J.Lange at al., "Charge collection studies of proton-irradiated n- and p-type epitaxial silicon detectors”, presented at the $11^{\text {th }}$ ESSD, Wildbad Kreuth, 2009, accepted in Nucl. Instr. Meth. A.

[13] L. Andricek et al., Trans. Nucl. Sci., Vol. 51 No.3 (2004), 1117.

[14] M. Beimforde et al., "Development of thin sensors and a novel inteconnection technology for the upgrade of the ATLAS pixel system”, Proceedings of the HSTD7 Symposium, Hiroshima (Japan), 2009.

[15] G. Pellegrini et al., Nucl. Instr. Meth. A 592 (1-2) (2008) 38-43.

[16] A. Zoboli, et al., Trans. Nucl. Sci., Vol. 55 No. 5, (2008), 2275-2284.

[17] M. Kohler, 15 ${ }^{\text {th }}$ RD50 Workshop, CERN, 2009.

[18] R. Bates, 15 ${ }^{\text {th }}$ RD50 Workshop, CERN, 2009.

[19] G. -F. Dalla Betta, “Characterization of 3D-DDTC detectors on -ptype substrates”,Conference Report of the IEEE NSS-MIC Conference, Orlando (USA), 2009.

[20] G. Kramberger et al., Nucl. Instr. Meth. A 609 (2009) 142. 